\title{
Image Evaluation of Apical Hypertrophic Cardiomyopathy in Octagenary: An Especial Report
}

\author{
Victor Rodrigues Ribeiro Ferreira, Maria Christiane Valeria Braga Braile-Sternieri, Eliana Migliorini \\ Mustafa, Sofia Braile Sabino, Cibele Olegario Vianna Queiroz, Bethina Canaroli Sbardellini, Giovanni \\ Braile Sternieri, Lucia Angelica Buffulin de Faria, Idiberto Jose Zotarelli Filho* and Domingo \\ Marcolino Braile \\ Domingo Braile Institute of Sao Jose do Rio Preto (SP), Brazil
}

\begin{abstract}
Received: 17 November, 2017; Accepted: 15 December, 2017; Published: 03 January, 2018
*Corresponding author: Idiberto Jose Zotarelli Filho, Domingo Braile Institute of Sao Jose do Rio Preto (SP), Brazil, Tel: + 55 (17) 3203-4039, +55(17) 8166-6537; E-mail: pesquisaclinica@institutodomingo braile.com.br or m.zotarelli@gmail.com
\end{abstract}

\begin{abstract}
Introduction: Yamaguchi Syndrome accounts for $15.0-25.0 \%$ of all cases of hypertrophic cardiomyopathies in Japan. In addition, about $54.0 \%$ of patients are symptomatic, including palpitations, dyspnoea, dizziness, syncope and chest pain.
\end{abstract}

Objective: To present a special case of Apical Hypertrophic Cardiomyopathy (AHCM) in a female and Brazilian patient over eighty years old, as well as their recovery with pharmacological treatment.

Highlight of Case: The present case report has its notorious importance due to its occurrence in patient without oriental descent; Longevity of the patient demonstrating the efficacy of pharmacological treatment; exhaustion of image evaluation methodologies for diagnosis and therapeutic conduction.

Conclusion: The evolution of imaging diagnostic resources has made AHCM evaluation more elaborate, often providing information that helps therapeutical conduction, allowing an increase in survival with a significant improvement in quality of life.

Keywords: Apical Hypertrophic Cardiomyopathy; Yamaguchi Syndrome; Brazilian Octagenary; pharmacological treatment

\section{Introduction}

Yamaguchi Syndrome represents 15.0 - $25.0 \%$ of all cases of hypertrophic cardiomyopathies in Japan [1]. Although it is the most prevalent of genetic diseases, the prevalence of AHCM in the world population is low, around 0.02 to $0.20 \%$. With initial studies supporting more benign prognosis but more recent reports suggesting cardiovascular morbidity from $25.0 \%$ to $30.0 \%$ and mortality in $4.0 \%$ to $29.0 \%$ of cases [2]. In addition, about $54.0 \%$ of the patients are symptomatic, including palpitations, dyspnoea, dizziness, syncope and chest pain [1,2].

Thus, AHCM is diagnosed by means of complementary examinations, where some alterations are detected: Echocardiogram ECG (T-wave and $>10.0 \mathrm{~mm}$ ); Echocardiogram (tip obstruction); Left ventriculography (aspect of swords [3]. Precordial pain is the most frequent symptom ranging from
$11.1 \% 1$ to $73.9 \%$; Following palpitations from 13.33 to $44.4 \%$; Dyspnea from 1337 to $33.3 \%$ and fatigue from 103 to $15.4 \%$ [3, 4].

Diagnosis is performed in adulthood, however, there are some severe cases in adolescence [5-9]. Sakamoto, et al. performed 171,306 electrocardiograms (ECG) in Japanese schoolchildren under 15 years of age, and found no case with $\mathrm{T}$ wave alterations [3]. The study by Morimoto, et al. in Japanese adults, who detected changes in giant $\mathrm{T}$ in 11 of $12,898(0,085 \%)$ males and in 6 of $21,232(0,028 \%)$ females, increasing the incidence with increasing age [16].

This was confirmed by Bielli, et al. who accompanied an 86-year-old patient, who had not presented any electrocardiographic changes until the age of 70 . The apical form can be transmitted genetically, as well as being associated with other types of AHCM in individuals of the same family [10].

The present study aimed to present a special case of apical hypertrophic cardiomiopathy in female and Brazilian patients over eighty years old, as well as their recovery with pharmacological treatment.

\section{Highlight of Case}

The present case report has its notorious importance due to its occurrence in a patient without oriental descent; Longevity of the patient demonstrating the efficacy of pharmacological treatment; exhaustion of image evaluation methodologies for diagnosis and therapeutic conduction.

\section{Case}

MHC, female, Caucasian, from Rio de Janeiro, with type 2 diabetes mellitus and hypothyroidism, began follow-up in 2012 with persistent dizziness and fatigue at medium efforts. The electrocardiogram revealed sinus tachycardia with an inverted $\mathrm{T}$ wave with a maximum amplitude of $6.00 \mathrm{~mm}$ and signs of Left Ventricular (LV) hypertrophy. Echocardiography revealed grade II diastolic dysfunction and preserved systolic function (Figures 
$1,2)$. LV analysis indicated significant hypertrophy in all apical segments, with normalization of mid-basal segment thickness. The aspect of the LV was noted in "swords suit", an image suggestive of Apical Hypertrophic Cardiomyopathy (AHCM). No significant gradient was identified in the left ventricle outflow tract, and the valvular apparatus evaluation identified mild, mild, mitral and tricuspid aortic regurgitation. Cardiac magnetic resonance revealed an increase in the thickness of all apical myocardial segments, being the largest apical-apical diameter $18.00 \mathrm{~mm}$ thick (Figure 3). It also revealed a discrete

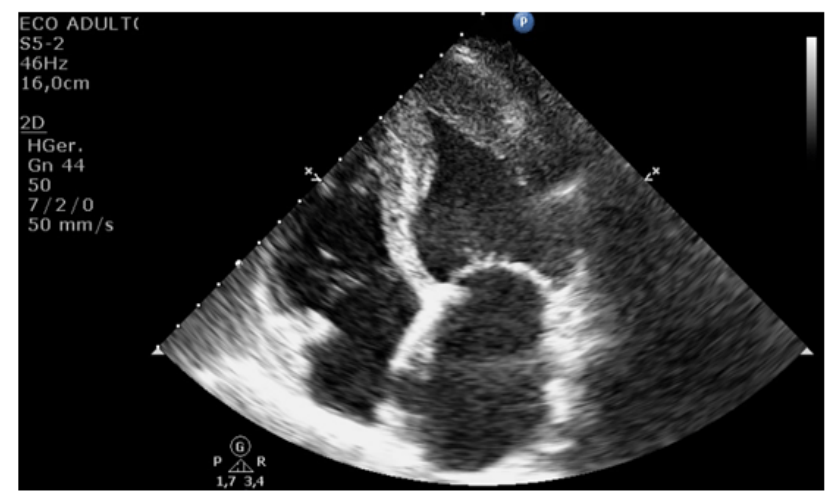

Figure 1: Diastole Echocardiography Image

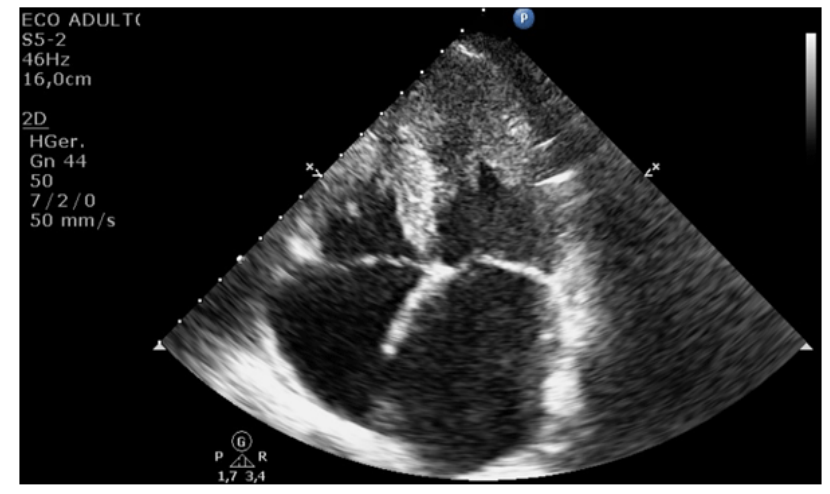

Figure 2: Systole Echocardiography Image

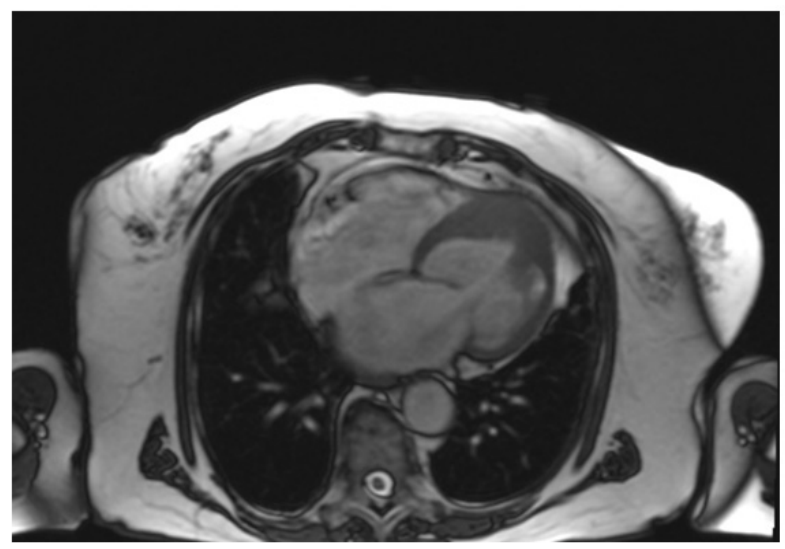

Figure 3: Resonance Magnetic Image area of late mesocardial enhancement, of non-coronary pattern in all apical segments, estimated in 2.0 grams, corresponding to approximately $2.0 \%$ of the myocardial mass. After four years of pharmacological treatment with amiodarone hydrochloride (100.0 mg/ day), trimetazidine dihydrochloride $(70.0 \mathrm{mg} /$ day), metoprolol tartrate $(50.0 \mathrm{mg} /$ day), acetylsalicylic acid $(100.0$ $\mathrm{mg}$ / day), rosuvastatin calcium $5.0 \mathrm{mg} /$ day) and levothyroxine sodium (100 mcg/ day). The patient continues with excellent clinical evolution and improved quality of life.

\section{Discussion}

The descriptions of AHCM date back to the 19th century. In 1979, Yamaguchi and his colleagues published a series of 30 patients with cardiomyopathy with apical presentation, a study that gained great repercussion and denominated Yamaguchi's Disease $[3,12]$.

Apical hypertrophic cardiomyopathy (Yamaguchi syndrome) is a rare subtype of hypertrophic cardiomyopathy. The syndrome is most common in Japan, where it was first described. Outside Japan, it is a very rare cause of hypertrophic cardiomyopathy $[12,13]$.

AHCM typically has an autosomal dominant inheritance pattern, but may also be sporadic. Recent studies suggest the association of AHCM with genetic mutations in ACTC1, TPM1, MYBPC3 and MYH7, and current studies are underway to identify other contributing genes. It is characterized by hypertrophy of the left ventricular apex, inversions of deep $T$ waves on the electrocardiogram, and a silhouette of ace-of-spades of the left ventricular chamber [1]. The phenotypic variants of AHCM include predominant apex hypertrophy, obstruction of the ventricular medium with cavity obliteration, and left ventricular aneurysm.

In this context, the heterogeneity of its presentation and clinical variability causes AHCM to have a late or lost diagnosis [1]. In the absence of epicardial coronary artery disease, AHCM is caused by myocardial ischemia due to the narrowing of the intramural coronary arteries of small vessels of the hypertrophied myocardium, as well as increased demand for oxygen in the myocardium [3]. Ventricular arrhythmias may also occur due to asymmetry hypertrophy of the left ventricle, resulting in dizziness, syncope or sudden cardiac death. Still, there is compromised filling of the left ventricle due to the stiffness of the wall and subsequent reduction of the cardiac output. Increased atrial pressures and dilatation of diastolic dysfunction can lead to atrial fibrillation, which predisposes to stroke [11].

Complementary imaging has become indispensable for the therapeutic planning of patients with AHCM [11]. In unusual presentations such as apical morphology, the determination of aspects such as myocardial fibrosis, valvar regurgitation, systolicdiastolic dysfunction, complex arrhythmias, myocardial ischemia, among others, shows the importance of diagnostic resources in order to allow longevity with quality of life as In the case described above [11-16].

It is important to differentiate this electrocardiographic alteration from others attributed to: Morgagni-Adams-Stokes syndrome, ischemic disease, bradycardia, metabolic disorder, 
right ventricular hypertrophy with right bundle branch block, encephalic changes and transient changes during coronary angiography due to apical hypertrophy [12].

Thus, only after the work performed by Yamaguchi et al., Covering 30 patients, it obtained a great repercussion, spreading the concept of this type of hypertrophic cardiomyopathy.

\section{Conclusion}

The evolution of imaging diagnostic resources has made AHCM evaluation more elaborate, often providing information that helps therapeutical conduction, allowing an increase in survival with a significant improvement in quality of life.

\section{Acknowledgements}

The work was supported by Domingo Braile Institute - Sao Jose do Rio Preto - SP, Brazil

\section{References}

1. Jorge Silva, Gerardo Palacios, Hector Luciardi. Apical hypertrophic cardiomyopathy. Yamaguchi syndrome. Rev Fed Arg Cardiol. 2016;45(4):208-209.

2. Jan MF, Todaro MC, Oreto L, Tajik AJ. Apical hypertrophic cardiomyopathy: Present status. Int J Cardiol. 2016 Nov 1;222:745759. doi: 10.1016/j.ijcard.2016.07.154

3. Sakamoto T, Tei C, Murayama M, Ichiyasu H, Hada Y. Giant T wave inversion as a manifestation of asymmetrical apical hypertrophy (AAH) of the left ventricle. Echocardiographic and ultrasonocardiotomographic study. Jpn Heart J. 1976;17(5):611-629.

4. Keren G, Belhassen B, Sherez J, Miller HI, Megidish R, Berenfeld D, et al. Apical hypertrophic cardiomyopathy: evaluation by non invasive and invasive techniques in 23 patients. Circulation.1985;71:45-56.

5. Steingo L, Dansky R, Pocock WA, Barlow JB. Apical hypertrophic non obstructive Cardiomyopathy. Am Heart J. 1982;104(3):635-637.

6. Kereiakes DJ, Anderson DJ, Crouse L, Chatterjee K. Apical hypertrophic Cardimiopathy. Am Heart J. 1983;105:855-856.
7. McDonnell MA, Tsagaris TJ. Recognition and diagnosis of apical hypertrophic cardiomyopathy. Chest. 1983; 84(5):644-647.

8. Vacek JL, Davis WR, Bellinger RL, Mckiernan TL. Apical hypertrophic cardiomyopathy in American patients. Am Heart J. 1984;108(6):1501-1506.

9. Sakamoto T, Hada Y, Amano K, Yamaguchi T, Takenaka K. Population study of mitral valve prolapse in the young: eight years experience. Circulation. 1984; 70(suppl 11):162-166.

10. Bielli M, Parravicini V, ZanettaM, Zenone F. Apical hypertróoica cardiomyopathy: description of an advanced age case with documentation of electrocardiographic evolution. G Ital Cardiol. 1991;21:1325-1329.

11. Pelliccia F, Pasceri V, Limongelli G, Autore C, Basso C, Corrado D, et al. Long-term outcome of nonobstructive versus obstructive hypertrophic cardiomyopathy: A systematic review and metaanalysis. Int J Cardiol. 2017;243:379-384. doi: 10.1016/j. ijcard.2017.06.071

12. Doctorian T, Mosley WJ, Do B. Apical Hypertrophic Cardiomyopathy: Case Report and Literature Review. Am J Case Rep. 2017;18:525528.

13. Candelario N, Penalver J, Sen M. Yamaguchi syndrome presenting as atrioventricular nodal re-entrant tachycardia in an AfricanAmerican patient. BMJ Case Rep. 2017;2017. pii: bcr2016218051. doi: 10.1136/bcr-2016-218051

14. Diaconu CC, Dumitru N, Fruntelata AG, Lacau S, Bartos D. Apical Hypertrophic Cardiomyopathy: The Ace-of-Spades as the Disease Card. Acta Cardiol Sin. 2015;31:83-86.

15. Klarich KW, Attenhofer Jost CH, Binder J, Connolly HM, Scott CG, Freeman WK, et al. Risk of death in long-term follow-up of patients with apical hypertrophic cardiomyopathy. Am J Cardiol. 2013;111(12):1784-1791. doi: 10.1016/j.amjcard.2013.02.040

16. Morimoto S, Osamura Y, Matsumura K, Harada M, Komatsu Y. On the incidence of giant negative $\mathrm{T}$ waves of the electrocardiograms among 34000 population and a cardiac study including ventriculography and endomyocardial biopsy. Resp Circul. 1981;29:1337-1340. 